\title{
A Framework for Contextualized Visualization Supporting Informal Learning
}

\author{
doi:10.3991/ijac.v2i3.996 \\ Eicke Godehardt \\ SAP Research, CEC Darmstadt, Germany
}

\begin{abstract}
The problem of the information society today is that knowledge workers are overwhelmed by the amount of information they get. This creates the need of a filter: the possibility to find relevant information in an easy and systematic way. In fact, working at a knowledge intensive workplace and retrieving information is a kind of learning informal learning - which takes place at daily work. This type of learning includes reading documents and digital snippets, asking questions and searching for the help of other colleagues.
\end{abstract}

This paper investigates how people at knowledge intensive workplaces can be supported through a graphical integration of existing information in a contextualized way to fulfill their given work task. We present research on software that visualizes the information of knowledge workers, tasks, digital resources, people and the relations between them. In addition state context information is taken to enrich the output to provide an intuitive and appropriate tool for knowledge workers. We will show all the necessary steps to offer these contextualized information in a supporting visualization.

To verify the usefulness of our approach, we did a quantitative and qualitative user study to see if contextualized information visualization is helpful to knowledge workers for a specific scenario. Therefore we analyzed how beginners or new employees may benefit from such a tool. The results clearly show the advantages of our solution. Contextualized visualization substantially boosts efficiency and effectiveness of knowledge workers, because of time savings and avoidance of failures.

Index Terms - Context Awareness, Visualization, Informal Learning, Knowledge-intensive Workplace.

\section{INTRODUCTION}

The main problem for knowledge workers today is to get the right information and data at the right time in a usable form. If information is delivered according to the current context (situation) of the knowledge worker, the content can be learned, assimilated and used in an effective manner.

Different studies show the valid demand and high requirements from knowledge workers, e.g., [1] and [2]. Especially the searching of information and collaboration partners are important key activities for informal learning at the workplace. In combination with task and process based learning and working, this is the main focus of the
European project called APOSDLE, which tries to merge the roles of learn, work and collaborate [3]. As a complement another project (EUDISMES) tries to offer process modeling techniques to end users [4]. Technically APOSDLE as well as EUDISMES are trying to use existing company data for informal learning.

Our hypothesis is that taking context information into account for knowledge or information visualization can boost the support of this kind of tools considerably, especially for beginners in a specific topic or working area, or completely new employees to get involved into wellestablished standard procedures and processes of that company.

The paper is structured as follows. After discussing the related work, we introduce our approach with a definition of context and a theoretically introduction of our framework. The prototypic implementation and instantiation of the framework is explained and presented afterwards. Subsequent the performed evaluation is discussed. We conclude with ideas for future work and a summary.

\section{RELATED WORK}

This work is related to three research areas. It is located in the intersection of the areas of informal learning at the workplace, knowledge visualization and contextualized systems.

As informal learning we follow the very general understanding as collected in [5]. Informal learning includes every unstructured learning, daily experiences and exposure to the environment - at home, at work, at play. Another important characteristic is the absence of a curriculum or other elements of formal education, like teachers and certificates. The importance of informal learning at knowledge intensive workplaces and especially for beginners in a new job surrounding is discussed in detail in [6] and [6]. Some of the elements or functions of informal learning they describe are interesting for our work, the information visualization: especially find information, skills and persons, get an overview and find relations between all data.

But there is little scientific work in the socio-technical intersection area with knowledge visualization and contextualized systems. So we will discuss two major related contributions.

The first one tries to adapt cooperative work spaces to the current work context of a distributed collaborating group of knowledge workers [8]. To accomplish this task, they define adaptation rules based on context information 
of the group to automatically adapt the user interface of the collaborative work space to the needs of the teams. But this approach only focuses on the user interface and not on relation context, the contextualization of information, which leads to new relations and associations.

The other interesting work can be found in [9]. The authors visualize relation context (information which could be derived from data sources, which is discussed below in more detail). Based on work context they try to choose the right content, with right detail, resolution, format, delivery time and data granularity.

But this work only introduced a conceptual framework without implementation and evaluation. In addition this approach is not modular, i.e., the visualization component is a fixed piece of the system. So it is not easily possible to add or change the kind of visualization or the visualization and placement algorithm. It is rather a system, with a fixed mapping of raw data to the visualization. Furthermore it is unclear if there is one or more external data sources and if contextualization can take part across data source boundaries. Another important point of this approach is the definition and understanding of the notion of "context". It is more related to cognitive processes and models, and it is unclear how this is technically perceived and realized. Additionally there is no evaluation based on there work.

We can conclude that there is still a lack of systems which enable contextualized information, based on desktop information and data relations to visually/graphically support knowledge workers at their workplace for informal learn and work processes.

\section{APPROACH}

To accomplish the demand of knowledge workers we propose the following solution. We developed a framework for contextualized visualization, which uses a combination of existing context information sensors, different knowledge visualizations and various data sources.

Our framework integrates different sources of information and data to enhance existing visualizations at the knowledge intensive workplace to support the knowledge workers.

\section{A. Context}

We have to define how we understand context. In general we follow the definition of Hartmann and Austaller [10]:

"Context characterizes the actual situation in which the application is used. This situation is determined by information which distinguishes the actual usage from others, in particular characteristics of the user (her location, task at hand, etc) and interfering physical or virtual objects (noise level, nearby resources etc). Thereby, we only refer to information as context that can actually be processed by an application (relevant information), but that is not mandatory for its normal functionality (auxiliary information)."

This means context can give us information about the current situation and the current work task of the knowledge worker. For further discussion we sub classify the term "context" into "relation context" and "state context". The former means relations in or between data sources and the latter information about the status of the user.
Definition relation context: "Relation context is the part of context information, which characterizes the environment respectively the relations of information or virtual objects among each other."

Relation context usually can be computed by analysis of the data (data mining). This is especially useful, if several data sources are combined [11]. Techniques to accomplish this task range from simple "pattern matching" over mechanisms from artificial intelligence to probabilistic approaches [12].

A concrete example could be the cross linking of authors of documents, which can be obtained from the metadata of the documents itself, and contact information from the mail tool of the user.

In contrast and complement we define state context as follows:

Definition state context: "State context is the part of context information, which describes the characteristics and properties of the user itself and his/her environment and cannot be extracted from static data or information."

Examples of state context information are geographical location of the user, her current work task or level of noise. All this information can help to adapt the workplace and especially the visualization for the better.

A central and significant source of this state context information is the operation system [13]. This provides mechanisms to get any kind of events and user interactions, like mouse movements and mouse clicks, but also keyboard events, clipboard content and information about open applications, windows, files and content of files. Fig. 1 shows how to hook into the operating system event or message stream.

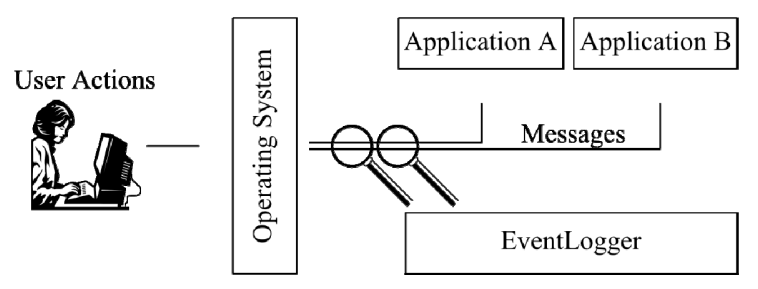

Figure 1. Contextualization approach

Some of this low level context information can be aggregated to higher level information, e.g., desktop topics or keywords based on file's and mail's content or the content of the current Internet page in the Internet browser of the user. Another example is the elicitation of the current work task. Machine learning methods are one possible medium to compute this information based on keyboard input, window titles and file content [13].

Other examples of context information are (physical) sensors like noise level and brightness. All this can be applied and integrated to optimize and adapt the visualization for the knowledge worker.

\section{B. Framework}

To fulfill this demand and provide the knowledge worker with a visualization, that fits his/her current situation and need, we developed a system for contextualized information visualization. Fig. 2 gives a general overview of this system we propose. 


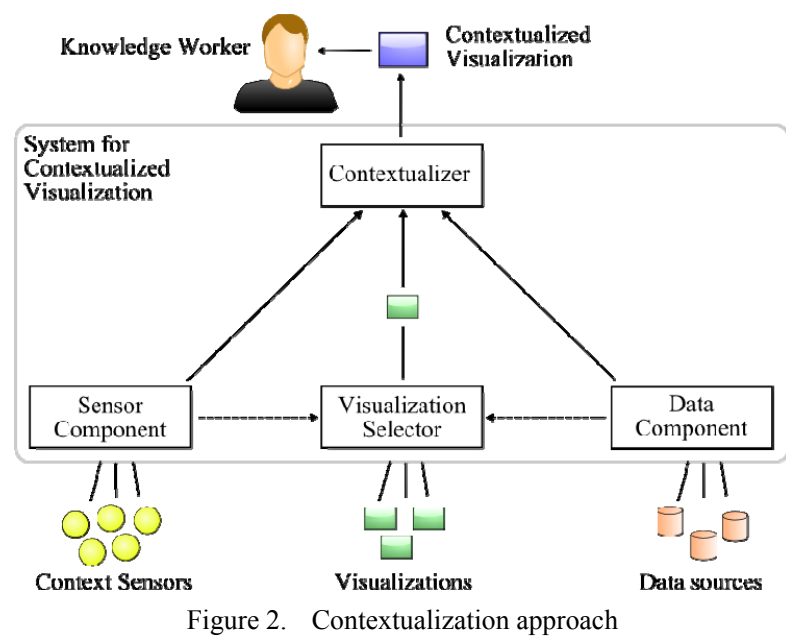

At the bottom of Fig. 2 there are three external elements we reuse for our framework:

- Context sensors (source of information about work context or the current work situation of the knowledge worker)

- Visualizations (existing adaptable knowledge or information visualizations)

- Data sources (at knowledge intensive work places this are usually tasks, processes and competency information and ontologies for domain concept definitions)

These three types of external elements are taken and combined in a new way, to support the knowledge worker's daily work.

Context information sources for our framework, which are useful to adapt the visualization according to the user's current working task include:

- Operating system based desktop sensor (can deliver information about active applications, open documents and files etc.)

- Desktop keywords based on user data (documents, clipboard and keyboard input etc.)

- User interactions and history

- Connections between elements of different data sources

- Type of data and data structure (like trees, nets and processes)

- User preferences

- Physical device information (like geographical location, screen size and bandwidth)

- Ambience information (e.g., noise level, luminosity)

Context sensor information can be of interest at launch time of the visualization, but also at runtime, if important context information changes. To meet this requirement, we use a push/pull mechanism to ask all context sensors at the beginning about all known information (pull). If context information changes during actual work, e.g., specific keyboard input or a new window appears, this information is forwarded to the visualization automatically (push). To ensure unobtrusiveness, the visualization itself has to prevent arbitrary redrawing with big visual differences.

On the data side, many data sources of any kind can be integrated, if an adapter is provided alongside. Usual kinds of data at knowledge intensive workplaces are: premodeled, implicit or ad-hoc processes, explicitly modeled domain information (usually by domain experts) or content management systems.

The last component in the center of the external elements at the bottom of Fig. 2 is the "Visualization Selector". Based on context information and data analysis, a fitting visualization is chosen. For the current implementation we did not investigate into this issue, but chose the visualization by the framework's configuration file or the user selects a visualization by herself.

The System supports a wide range of visualization types. By implementing a simple interface, additional visualizations may be added later in an easy way. This is true for any of the external components. Data sources as well as Context Sensors can be added at any time in a simple manner implementing a sensor interface or visualization interface respectively.

At the final step the contextualization is done. Context information is taken to adapt the visualization. This final step will apply the context information (describing the user's current situation) to a chosen visualization and data set.

Some examples of context-based adaptable visualization parameters are:

- highlight important or helpful information, based on desktop keywords, e.g., typed by the users or extracted from open documents (the importance of keywords may be estimated, e.g., by matching with explicitly defined user models [13])

- choose automatic starting point of visualization, e.g., current work context or task

- size and scaling of whole visualization or single elements according to available space or screen resolution

- adjustment of single visual elements, e.g., status of tasks or persons, or size of icons to show importance or complexity

- adjust level of detail for a given work context

Below we will describe concrete contextualization options for the graphical realization of these visualization parameters.

The result of the overall process is a visualization of important data, with the right amount of information, with the right level of detail, which best fits to the current work context or work situation of the knowledge worker.

\section{PROTOTYPIC IMPLEMENTATION}

The prototypic implementation of the above introduced framework was done in Java, to allow easy extension of the system with new context sensors, data sources and visualizations.

For a scenario to instantiate the framework we used the research project EUDISMES [4]. EUDISMES means "End User Development In Small and Medium Enterprise Software systems". It offers a collaborative task management component CTM. This tool integrates into MS Outlook to enable the users to define ad-hoc processes just by defining new tasks, sub-divide and delegate them.

Every task has a corresponding person, the owner of the task. In addition to this relation from task to the corresponding person (responsible person), there can also be 
relations to helpful or necessary documents of any kind. These documents are associated or attached by the user while creating or working on the given task. If such an adhoc process seems to be valuable, it can be used as a template for new processes, with all the persons and documents involved.

Fig. 3 shows a screenshot of the resulting application. On the left side is the information visualization of a net of different kinds of data. In separate sectors, hierarchies of tasks (ad-hoc processes), a network of people, related to these tasks, and associated documents of any type are shown together with the relationship. The user may adjust the size of any of the sectors for focus on her topic of interest. The most interesting part of this visualization besides the relationships is the adaptation and contextualization of the elements.

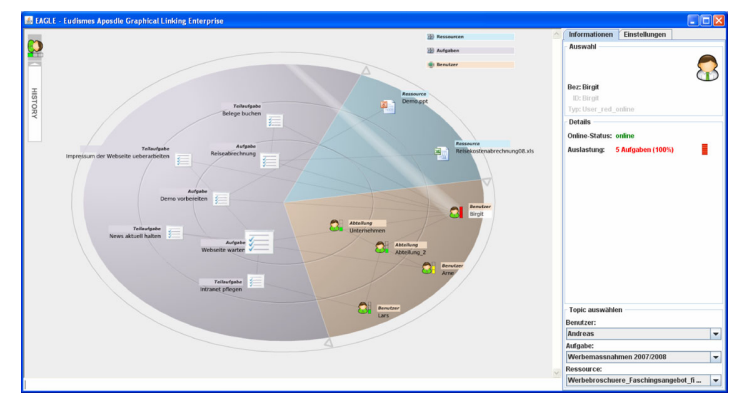

Figure 3. Prototype

In addition to the graphical visualization of the data the application has a textual information space showing more and deeper data on the right side. It shows extra information regarding the selected item, like due date and status of tasks, workload in number of tasks for any of the visualized people. This is information which is not easily embeddable into the graphical visualization. The sidebar also allows another way of navigation through the data by offering drop down lists for all elements.

At the end this tool allows the knowledge worker to navigate through the displayed information and explore the relationship of responsive people to task, importance of tasks, availability and workload of people and associated documents for tasks.

\section{A. Context information}

The application is analyzing the information from the CTM tool, to find all the inter relationships between tasks, persons and documents. In addition context information are computed based on heuristics, which allows emphasizing tasks regarding there due time or complexity (number of sub-tasks and delegations) and persons regarding there workload (number of associated tasks). Supplementary status context data is taken from desktop sensors or other external tools (see above). Some possibilities are shown in Fig. 4. On the left side all supported combination availability status and work load is shown. To set the level of $100 \%$ work load, we take the person with the highest number of tasks. The range for yellow a marking is from $30-70 \%$ according to this highly engaged person. On the right side the task's complexity is visualized as the size of the graphical element. All this are heuristics, but may improve the usefulness for the knowledge worker.

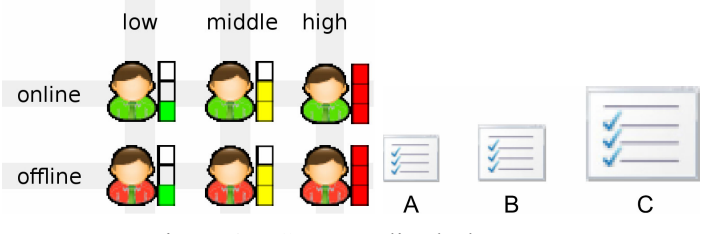

Figure 4. Contextualized Elements

The context information is partly embedded into the visualization and partly into the sidebar, depending on type of context and importance. The following list gives an overview about all supported context information together with its visual position realized in this instantiation of our framework (partly shown in Fig. 4):

- complexity of task: size of icon in visualization

- due date of task: colored bar and number of days as text below task icon in sidebar

- availability of person: color of icon in visualization

- workload of person: colored bar beneath the person and as number of tasks as text in sidebar

This context information and especially its visualization or parameterization of the visualization allows an easy overview and perception of information by the knowledge worker at her desk.

For state context information a context sensor based on information of the operating system or special tools are needed. For example availability of other persons may be obtained out of an instant messaging tool.

\section{B. Chosen visualization}

As the visualization we choose the TopicMap Viewer [14]. This visualization was chosen due to the following facts and advantages. One major point is that the visualization is explicitly developed with tree structured or hierarchical data in mind.

It also offers a great flexibility, like adaptable graphical icons and dynamic configuration and is adjustable in regards to many (graphical) options.

Another important issue is usability. The TopicMap Viewer is highly usable and allows the user to navigate through the data in a fast and easy way. To prevent cognitive overload, it only shows part of the data based on the selected information to automatically reduce the amount of visible information.

To support the user while browsing through huge data sources, a history option is provided. Another important issue is figure consistency, which gives orientation to the user. This consistency is achieved by minimize shifting of items and sectors. So usually, if the user comes back to a topic already visited, the graphical appearance would be the very same.

In summary the main advantage of TMV is, that it displays a large quantity of information with specific features like zooming the required activity, hiding the inactive data without losing track of links with other topics which are currently not required and differentiates the topics according to types by using sectors and levels.

\section{Evaluation}

To verify the usefulness and relevance of our approach, we did a quantitative and qualitative user study. The evaluation was done in two dimensions: quality (feeling 
and opinion of the user), efficiency (time the user needs to fulfill the given task) and effectiveness (number of failures). To measure results in both dimensions we used a questionnaire for quality evaluation and time performance test based on three tasks a user had to fulfill for quantitative data.

\section{A. Evaluation Setup}

In this section we would like to introduce the evaluation setup or how we designed the user study.

To find a representative user group, we choose 30 people for our user study. All users had academic background (students, PhDs or other scientific staff members) to get a representative sample for our target group - the knowledge worker.

We chose a kind of cross evaluation. That means a combination of between-groups and within-groups evaluation design. This will give us the advantages from both designs: smaller user groups (from within-groups) while making sure, that other effects (like learning in the time of the study) are limited or eliminated (from between groups).

For the user groups that means we split the whole group of 30 into two groups of 15 people. On the other hand, every attendant from both groups had to fulfill the tasks twice - once with our contextualized visualization system and once without. To eliminate or at least realize learning effects, the order was interchanged, so 15 users started with our system (scenario A) and the other 15 started without the contextualized visualization system (scenario B). This way we could see learning effects in the measured times if they occur.

As every user ran through both scenarios (with and without our system), we changed the tasks slightly for both scenarios. This way, we tried to avoid, that a user remembers the right answer from the first run and simply answer from memory in the second run. The tasks which had to be dealt with where:

1. Find the corresponding person for a given job

2. Delegate a job to a person with high capacity (lowest workload)

3. Find a given document

For the study itself, a knowledge intensive workplace was simulated. All users got printed documentation. Our prototype was already running on the computer. MS Excel was used to semi-automatically measure the times. For the second scenario (without the contextualized visualization system) a telephone was also simulated. The attendant could ask questions to the supervisor of the experiment, by telling the name of the person to contact and a question to ask. To adjust this a little bit, we add a penalty of 10 seconds for the "telephone calls" to simulate real calling or walking to the next office. 10 seconds is a small assumption, especially when concerning small talk, which usually is taking place in such situations, or finding a person in a slightly farther office or department.

\section{B. Time Measures}

In Table 1 the average times of the three tasks are shown, separately for the two scenarios. Scenario A means with support of the contextualized visualization system and Scenario B is without this kind of support.
For task 1 and 2 the average duration is clearly shorter when using the supporting graphical system. But also after deeper analysis of the 30 users for all tasks, the result clearly shows the benefit of the system.

For the deeper analysis we choose a t-Test, which is designed to compare different sets of samples with each other. Table 1 also shows the t-Test values for the three tasks (4.0403 and 8.2402 for tasks 1 and 2). This indicates that the results are highly significant (for the significance level $\alpha$ of $0.01 \%$ the threshold for a pared test would be 4.011). As both numbers exceed this threshold, it shows the high validity and high significance of our results.

TABLE I.

AVERAGE TIME FOR TASKS AND SCENARIOS

\begin{tabular}{|c|c|c|c|}
\cline { 2 - 4 } \multicolumn{1}{c|}{} & Task 1 & Task 2 & Task 3 \\
\hline Scenario A & $\mathbf{5 7}$ sec. & 33 sec. & 51 sec. \\
\hline Scenario B & $\mathbf{8 7}$ sec. & $\mathbf{9 7}$ sec. & 39 sec. \\
\hline t-Test Value & $\mathbf{4 . 0 4 0 3}$ & $\mathbf{8 . 2 4 0 2}$ & $\mathbf{- 2 . 0 7 1 5}$ \\
\hline
\end{tabular}

Our observations regarding task 3 are different. Here the results are opposite. A potential explanation for this result is the following one. As already outlined above, task 3 was to find a document. The experimental setup was probably too unusual for a knowledge intensive workplace. The prepared file hierarchy was pretty easy to follow and the requested document was the only one in the target folder. Perhaps this does not reflect a real workplace, where hundreds or thousands of documents reside in a sometimes unclear hierarchy. So the last task may not be representative for a real knowledge worker's environment.

The quantitative results further show, that the learning effects had no big influence on the measured times. On the other hand the number of errors made by the attendants really differs, whether they got contextualized support or not. Though the number is not representative, our system improves the effectiveness.

\section{Quality Results}

In addition to the quantitative analysis based on time measures, a quality evaluation complements this user study based on a questionnaire.

This analysis and interpretation of the questionnaire is about the user satisfaction. The questionnaire itself is partly based on ISO (national standardization committee) standard ISO 9241-151:2008-09 for user interface and usability evaluations. It contains 13 questions, while 11 are multiple choice and two free text questions to get annotations and comments from the users.

To enumerate each and every question together with all answers would break the size of this paper. Instead we are going to present the main and key facts and results from the questionnaire.

First and foremost, all users found the system at least helpful if not, as most users did (63\%), very helpful. Most attendants said that the system is self explanatory and almost all attest unobtrusiveness to the contextualized visualization. Contextualized information is explicitly judged as supportive for the given tasks. 
The question about which task was best and which task was least supported by the system, the answers where as estimated. As the numbers above already indicated, most users found it least helpful for task 3 (discussed in detail above), but very helpful for task 1 and especially task 2 .

All in all, the evaluation strongly confirms the importance and potential of our approach.

\section{FUTURE WORK}

There are a number of interesting context sensors which could be integrated into our framework to further extend the usefulness of this approach for knowledge intensive workplaces. For example application based tracking of low level user interactions or the very promising above explained desktop keyword utilization. The latter is the context monitor daemon, for topic detection based on currently open documents and user typing, and is directly related to the presented scenario.

We also think of combining this prototype with other experiments and additional visualizations to broaden the field of application. To clarify the evaluation results regarding task 3, we will enhance our measurements by a systematic comparison with more realistic file hierarchies.

\section{SUMMARY}

In this paper we presented the design, prototypic implementation and evaluation of a framework for contextualized visualization at knowledge intensive workplaces. By using a combination of (already existing) context sensors, visualizations and data sources, we built a prototype that offers great visual support to knowledge workers.

The evaluation showed a significant improvement by our approach to knowledge intensive work. This holds especially for informal learning, which among other things, means information finding and task/cooperation based activities. In particular, our system helps knowledge workers to get familiar with tasks and processes in a new environment or working area. We showed that at least some tasks at knowledge intensive workplace clearly benefit from our approach.

\section{ACKNOWLEDGMENT}

APOSDLE is partially funded under the FP6 of the European Commission within the IST work program 2004 (FP6-IST-2004-027023). EUDISMES is a research project partially funded by the German Federal Ministry of Education and Research.

\section{REFERENCES}

[1] Kathryn Rudie Harrigan and Gaurav Dalmia: Knowledge Workers: The Last Bastion of Competitive Advantage. In: Strategy \& Leadership 19, 1991.
[2] Peter Ferdinand Drucker: The New Society of Organizations; In: Harvard Business Review 70, 1992.

[3] Stefanie N. Lindstaedt and Tobias Ley and Harald Mayer: Integrating Working and Learning with APOSDLE. In: Proceedings of the 11th Business Meeting of Forum Neue Medien, Vienna, 2005

[4] Todor Stoitsev and Stefan Scheidl and Michael Spahn: A Framework for Light-Weight Composition and Management of Ad-Hoc Business Processes, LECTURE NOTES IN COMPUTER SCIENCE, Springer, 2007

[5] Alan Rogers: Looking again at non-formal and informal education - towards a new paradigm. In: The encyclopaedia of informal education, 2004.

[6] Michael Eraut: Non-formal learning, implicit learning and tacit knowledge in professional work.. In: The Necessity of Informal Learning (2000), pp. 12-31

[7] P. Robert-Jan Simons: Towards a constructivistic theory of selfdirected learning. In: Conceptions of Self-Directed Learning: Theoretical and Conceptional Considerations/LOS, Learning Organized Self-Directed Researchgroup, pp. 155-169, 2000.

[8] Dirk Veiel, Joerg Hake and Stephan Lukosch: Context-based Adaptation of Cooperative Work Spaces (original in German: Kontextbasierte Adaption kooperativer Arbeitsbereiche). In: HEGERING, Heinz-Gerd (Hrsg.); LEHMANN, Axel (Hrsg.); OHLBACH, Hans J. (Hrsg.); SCHEIDELER, Christian (Hrsg.): GI Jahrestagung (2) Bd. 134, GI, 2008 (LNI). - ISBN 978-388579-228-4, 808-814

[9] Eui-Chul Jung and Keiichi Sato: A Framework of Context- Sensitive Visualization for User-Centered Interactive Systems. In: Proceedings of the $10^{\text {th }}$ International Conference on User Modeling (UM 2005)(Edinburgh, Scotland, UK, July 24-29, 2005), Lecture Notes in Computer Science 3538 (2005), S. 423-427

[10] Melanie Hartmann and Gerhard Austaller: Context Models and Context-awareness. In: Ubiquitous Computing Technology for Real Time Enterprises, p. 235-256, 2008, IGI Publishing

[11] Gregory Brian Judelman: Knowledge Visualization, Problems and Principles for Mapping the Knowledge Space; International School of New Media, University of Lübeck, 2004

[12] Natalya F. Noy: Semantic integration: a survey of ontology-based approaches; In: ACM SIGMOD Record 33, Number 4, 2004, p. 65-70 (doi:10.1145/1041410.1041421)

[13] Robert Lokaiczyk, Andreas Faatz, Arne Beckhaus, and Manuel Goertz. Enhancing Just-in-Time E-Learning through Machine Learning on Desktop Context Sensors. In: Proceedings of CONTEXT ‘07, 2007, 330-341.

[14] Eicke Godehardt and Nadeem Bhatti: Using Topic Maps for Visually Exploring Various Data Sources in a Web-Based Environment. In Scaling Topic Maps: Third international Conference on Topic Maps Research and Applications, TMRA 2007 Leipzig, Germany, October 11-12, 2007 Revised Selected Papers, Springer

\section{AUTHORS}

Eicke Godehardt is with SAP Research CEC Darmstadt, Bleichstr. 8, 64283 Darmstadt, Germany (e-mail: eicke.godehardt@sap.com).

This article was modified from a presentation at the ICELW 2009 conference in New York, NY, USA, June 2009. Submitted, July 4, 2009. Published as resubmitted by the author(s) on July 4 , 2009. This work was supported in part by the European Commission and the German Government. 